\title{
Oral Findings and Craniofacial Morphology in a Patient with Schwartz-Jampel Syndrome and Severe Obstructive Sleep Apnea: A Case Report
}

\author{
Mevadee Pibulniyom $^{1}$, Patimaporn Pungchanchaikul ${ }^{2}$, Thanate Assawakawintip ${ }^{3}$, and \\ Supakit Peanchitlertkajorn ${ }^{3}$ \\ ${ }^{1}$ Srinakharinwirot University Panyananthaphikkhu Chonprathan Medical Center \\ ${ }^{2}$ Khonkaen University Faculty of Dentistry \\ ${ }^{3}$ Mahidol University Faculty of Dentistry
}

May 14, 2020

\begin{abstract}
This is a report of an 8-year-old male with Schwartz-Jampel syndrome with severe obstructive sleep apnea. Dental findings include severe maxillary crowding, posterior crossbite, missing lower incisors, and supernumerary lower premolar. Significant craniofacial characteristics include Type I skeletal relationship with bimaxillary hypoplasia, hyperdivergent skeletal pattern, severe constriction of dental arches.
\end{abstract}

\section{Keywords}

Schwartz-Jampel syndrome, Pediatric Obstructive Sleep Apnea, Bimaxillary Hypoplasia, Posterior Crossbite, Case Report

\section{Key Clinical Message}

We reported dental and craniofacial characteristics of a patient with SJS and severe OSA. These findings demonstrate that children with SJS could be affected not only on general physical features but also craniofacial development.

\section{Introduction}

Schwartz-Jampel Syndrome (SJS1, ORPHA:800; OMIM\#255800) is a very rare genetic disorder with prevalence less than 1:1,000,000 ${ }^{1}$. SJS, named after Schwartz and Jampel $(1962)^{2}$, is an autosomal recessive disorder caused by mutations in HSPG2 gene. This gene encodes for perlecan, a cellular matrix protein regulating muscle contraction and chondrogenesis. ${ }^{3}$ A defective HSPG2 gene results in prominent SJS features of prolonged muscle contraction (myotonia) and multiple skeletal abnormalities (chondrodysplasia). Facial dysmorphology is distinctive and rather consistent across ethnicities. ${ }^{4}$ There are approximately 130 SJS reports in the current literature with only a few describing dental findings. Díaz-Serrano et al ${ }^{5}$ delineated oral manifestations such as dental crowding, high-arched palate, multiple impacted teeth and TMJ problem found in two siblings with SJS. Mallineniet al ${ }^{6}$ reported a Chinese boy with SJS who presented with bulbous crowns with marked cervical constriction, amber-brown discoloration, brittle enamel, soft dentine, and severe attrition in his primary teeth. A macrodontic primary incisor and a congenitally missing permanent incisor were also described in the same patient. ${ }^{6}$ In this paper, we present an 8-year-old male patient with SJS and severe obstructive sleep apnea (OSA). To the best our knowledge, this is second SJS case with OSA 
reported in the literature after Cook and Borkowski. ${ }^{7}$ In addition to oral findings, a craniofacial morphology of this patient will be presented.

\section{Case presentation}

An 8-year-9-month-old male patient of normal intelligence with SJS was referred to the Department of Orthodontics, Faculty of Dentistry, Mahidol University, Thailand. He is the only child of a healthy nonconsanguineous Thai couple. His birthweight was $3.070 \mathrm{~kg}$ with unremarkable prenatal history. Generalized muscle stiffness and abnormal gait have been observed since his first year. The diagnosis based on SJS's cardinal signs, was made at 3 years of age. His medical history includes hypothyroidism, repeated hospitalization due to severe allergy and respiratory distress, and severe OSA. He regularly receives carbamazepine to relieve his muscle contraction, and levothyroxine for hypothyroidism. Parents noticed occasional snoring at 6 months old and became worsened with increasing age. Other nocturnal symptoms included enuresis, choking, and gasping for air. Daytime symptoms include frequent morning drowsiness and headache, nausea, hypersomnolence, as well as poor concentration. He was referred to a sleep physician for a diagnostic polysomnography at the age of seven. The diagnosis for severe OSA was confirmed with an apnea-hypopnea index of 29 events/hour. The continuous positive airway pressure (CPAP) was prescribed instead of adenotonsillectomy to avoid a potential risk of malignant hyperthermia during a general anesthesia.

On physical examination, the patient had a proportionate short stature with his weight in the tenth percentile. He presented with multiple skeletal deformities, and generalized muscle stiffness. Extra-oral examination revealed a distinctive mask-like facie, blepharophimosis, low-set ears, microstomia and pursed lip, limited mouth opening $(25 \mathrm{~mm})$, asymmetrical oval facial form, convex facial profile and bimaxillary retrognathia (Fig. 1). On oral examination, the patient was in mixed dentition with very severe dental crowding especially in the upper arch. The amount of dental crowding was measured at 12 and $6 \mathrm{~mm}$ for the upper and lower arch respectively. There was untreated active caries noted. The maxillary arch was severely constricted with a very high-vaulted palate. The upper intermolar width was significantly reduced with a measurement of $36 \mathrm{~mm}$. Bilateral posterior crossbite was observed (Fig. 1). Dental development was within normal range. Permanent mandibular lateral incisors were clinically missing. Enlarged palatine tonsils were present in the retropharyngeal area. Radiographic examination confirmed congenital hypodontia of the lower laterals and supernumerary mandibular teeth at the premolar region bilaterally (Fig. 2). Cephalometric analysis demonstrated that the patient had Type I skeleton relationship with retrognathic maxilla and mandible. The mandible rotated posteriorly while the maxilla exhibited counter-clockwise rotation. This creates a hyperdivergent pattern of the jaw bones. Both upper and lower incisors are retroclined and retruded. Soft tissue measurements showed the upper and lower lip were in a retrusive position following the underlying skeletons. The detailed cephalometric characteristics of the patient is presented in Table 1.

\section{Discussion}

Dental crowding is usually a result of the discrepancy between tooth and jaw size. In this case report, the severe dental crowding was likely a result of constricted jaw bones rather than larger teeth since the patient exhibited very narrow maxillary and mandibular arches. This also demonstrates that SJS could have effects not only on general bones but also craniofacial skeletons. Good oral hygiene helps prevent premature loss of primary teeth that could worsen the arch length discrepancy. Therefore, routine dental care and regular dental check-up should be emphasized. The patient's maxilla is constricted anteriorly and even more so laterally. The mandible is also retrognathic. This could illustrate the effects of myotonia on craniofacial development. Because maxillary and mandibular hypoplasia could be a risk factor in developing pediatric OSA. ${ }^{8}$ This could mean that patients with SJS are at risks to develop OSA as the patient in this report. However, this correlation has not been previously raised, possibly due to the rarity of SJS. This is the first case report of SJS from Thailand. It is also the first SJS report with a detailed description of craniofacial morphology.

\section{Conclusion}

SJS is a rare genetic syndrome with only a few reports of oral findings in the literature. The patient presented 
with typical features of SJS includes myotonia, short structure, abnormal gait, mask-like facie, blepharophimosis, microstomia, retrognathia and pursed lip. In addition, he was also diagnosed with a severe Obstructive Sleep Apnea which affected his daily activities and quality of life. We reported his oral findings that included severe dental crowding and narrow maxillary and mandibular arches. This is also the first study describing detailed craniofacial characteristics of a patient with SJS. The clinical findings of this patient suggest that SJS could have effects not only on general but also on craniofacial skeletons. And myotonia of craniofacial muscles could results in a diminished maxillomandibular development. Since maxillary and mandibular hypoplasia is a risk factor for pediatric OSA. patients with SJS could be at risk to develop OSA as the patient in this report.

\section{Authorship List:}

Supakit Peanchitlertkajorn conceived the ideas and supervised the treatment;

Thanate Assawakawintip, and Supakit Peanchitlertkajorn collected the data;

Supakit Peanchitlertkajorn, Mevadee Pibulniyom, and Patimaporn Pungchanchaikul analysed the data;

Supakit Peanchitlertkajorn, Mevadee Pibulniyom, and Patimaporn Pungchanchaikul led the writing.

\section{Reference}

1. FONTAINE B. Orphanet: Schwartz Jampel syndrome. Orphanet. https://www.orpha.net/consor/cgibin/OC_Exp.php?Lng=GB\&Expert=800. Published 2020. Updated February 2020. Accessed 02, April 2020.

2. SCHWARTZ O, JAMPEL RS. Congenital Blepharophimosis Associated with a Unique Generalized Myopathy. Archives of Ophthalmology.1962;68(1):52-57.

3. Nicole S, Davoine C-S, Topaloglu H, et al. Perlecan, the major proteoglycan of basement membranes, is altered in patients with Schwartz-Jampel syndrome (chondrodystrophic myotonia). Nature Genetics. 2000;26(4):480-483.

4. Viljoen D, Beighton P. Schwartz-Jampel syndrome (chondrodystrophic myotonia). J Med Genet. 1992;29(1):58-62.

5. Díaz-Serrano KV, Brandão CB, Brandão RB, Watanabe PCA, Regalo SCH. Dental findings and muscularskeletal features in Schwartz-Jampel syndrome: case report of two affected siblings. Spec Care Dentist. 2006;26(5):225-229.

6. Mallineni SK, Yiu CKY, King NM. Schwartz-Jampel syndrome: a review of the literature and case report. Special Care in Dentistry.2012;32(3):105-111.

7. Cook SP, Borkowski WJ. Obstructive Sleep Apnea in Schwartz-Jampel Syndrome. Archives of Otolaryngology-Head \& Neck Surgery.1997;123(12):1348-1350.

8. Guilleminault C, Huang Y-S. From oral facial dysfunction to dysmorphism and the onset of pediatric OSA. Sleep Medicine Reviews. 2018;40:203-214.

\begin{tabular}{|c|c|c|c|c|c|}
\hline & Cephalometric measurements & Norm & SD & Pre- treatment & Interpretation \\
\hline \multirow[t]{8}{*}{ Skeletal } & Antero-posterior: & & & & \\
\hline & SNA & 84 & 3.58 & 78.5 & Retrognathic maxilla \\
\hline & SNB & 81 & 3.59 & 77 & Retrognathic mandible \\
\hline & $\mathrm{ANB}$ & 3 & 2.50 & 1.5 & Skeletal type I \\
\hline & A to $\mathrm{N}$ perpend & 4.59 & 3.86 & -5 & Retrognathic maxilla \\
\hline & Pg to N perpend & 0.47 & 5.96 & -1.1 & Orthognathic mandible \\
\hline & $\begin{array}{l}\text { Vertical: } \\
\text { NS-MP }\end{array}$ & 30 & 5.61 & 39 & Posterior rotation of mandi \\
\hline & NS-PP & 9 & 3.03 & -1 & Anterior rotation of palatal plane \\
\hline
\end{tabular}




\begin{tabular}{llllll}
\hline & Cephalometric measurements & Norm & SD & Pre- treatment & Interpretation \\
\hline \multirow{3}{*}{ Dental } & MP-PP & 21 & 5.25 & 40 & Open configuration \\
& Antero-posterior: & & & & \\
& U1-NA & 22 & 5.94 & 6.5 & Retroclined U1 \\
& U1-NA (mm) & 5 & 2.13 & -1 & Retruded position of U1 \\
& L1-NB & 30 & 5.61 & 9 & Retroclined L1 \\
\multirow{5}{*}{ Soft tissue } & L1-NB (mm) & 7 & 2.22 & 0 & Retruded position of L1 \\
& H-angle & 14 & 3.83 & 5 & Retruded position of upper lip \\
& Lower lip to E-plane & 2 & 2.03 & -2 & Retruded position of lower lip \\
\hline
\end{tabular}

Table 1 Cephalometric measurements of the patient with SJS

Fig.1 Extraoral and intraoral pictures. Facial frontal (a), Facial profile (b), Maxillary occlusal (c), Mandibular occlusal (d), Right occlusion (e), Frontal occlusion (f), and Left occlusion (g)

Fig. 2 Cephalometric (a) and Panoramic radiographs (b) of the patient.
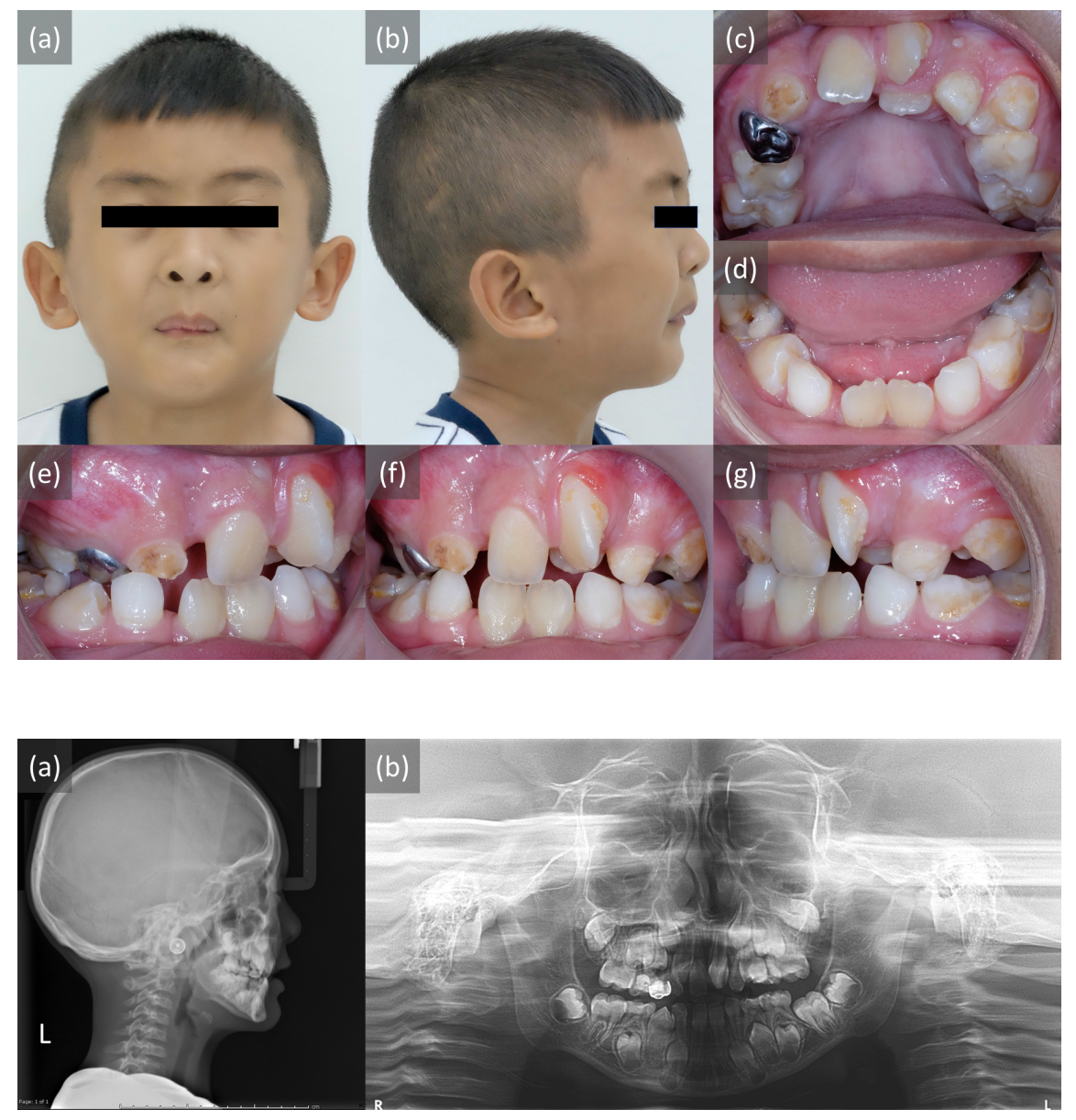